\title{
The effect of alkaline treatment on tensile properties of sugar palm fibre reinforced epoxy composites
}

\begin{abstract}
A study on the effect of alkaline treatment on tensile properties of sugar palm fibre reinforced epoxy composites is presented in this paper. The treatment was carried out using sodium hydroxide $(\mathrm{NaOH})$ solutions at two different concentrations and three different soaking times. The hydrophilic nature of sugar palm fibre makes it difficult to adhere to hydrophobic epoxy and therefore posed the problem of interfacial bonding between fibre and matrix and such treatment was needed to alleviate such problem. The composite specimens were tested for tensile property determination. Some fractured specimens were examined under scanning electron microscope (SEM) to study the microstructure of the materials. Inconsistent results were obtained for tensile strengths, which indicate that the treatment is not very effective yet to improve the interfacial bonding. However, for tensile modulus, the results are much higher than untreated fibre composite specimens, which proved the effectiveness of the treatment.
\end{abstract}

Keyword: Arenga pinnata; Sugar palm fibre; Epoxy composites; Alkaline treatment; Tensile properties 